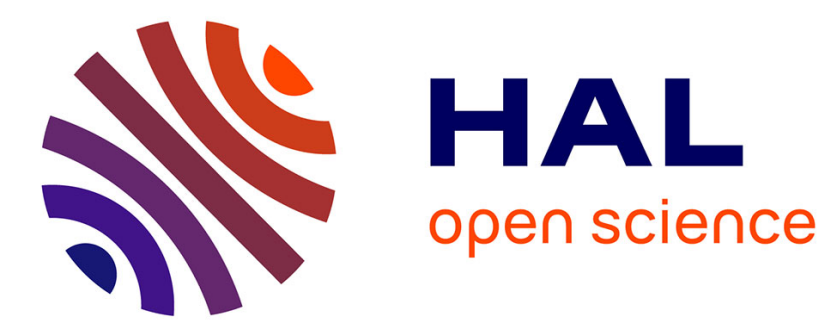

\title{
Optimal PMU-based monitoring architecture design for power systems
}

Didier Georges

\section{To cite this version:}

Didier Georges. Optimal PMU-based monitoring architecture design for power systems. Control Engineering Practice, 2013, 10.1016/j.conengprac.2013.11.019 . hal-00938362

\section{HAL Id: hal-00938362 https://hal.science/hal-00938362}

Submitted on 29 Jan 2014

HAL is a multi-disciplinary open access archive for the deposit and dissemination of scientific research documents, whether they are published or not. The documents may come from teaching and research institutions in France or abroad, or from public or private research centers.
L'archive ouverte pluridisciplinaire HAL, est destinée au dépôt et à la diffusion de documents scientifiques de niveau recherche, publiés ou non, émanant des établissements d'enseignement et de recherche français ou étrangers, des laboratoires publics ou privés. 


\title{
Optimal PMU-Based Monitoring Architecture Design for Power Systems ${ }^{1}$
}

\author{
Didier Georges ${ }^{\mathrm{a}}$ \\ ${ }^{a}$ Grenoble INP / Univ Grenoble 1 / Univ Grenoble 3 / CNRS, GIPSA-lab, F-38402 \\ Saint Martin d'Hères, \\ (e-mail: didier.georges@gipsa-lab.grenoble-inp.fr, phone: +33476826236, fax: \\ +33476826388).
}

\begin{abstract}
This paper is dedicated to a new methodology for designing an optimal monitoring architecture by using a limited number of PMUs (Phasor Measurement Units) and PDCs (Phase Data Concentrators). The optimal design problem consists in defining the optimal location of both PMUs and PDCs by maximizing the expected value of the trace of the observability gramian of the power system over a large number of set point scenarios, while minimizing some communication infrastructure costs. Furthermore, a nonlinear dynamical state-observer, based on the Extended Kalman Filter, is proposed. This state-observer allows to take transient phenomena into account for wide-area power systems described by algebraic-differential equations, without needing nonlinear inversion techniques. The overall approach is illustrated with the IEEE 10 generator 39 bus New England power system.
\end{abstract}

Keywords: Power system monitoring, optimal design of monitoring achitecture, sensor networks, observability gramian, optimal location of PMUs and PDCs, dynamical state estimation, extended Kalman filtering.

\section{Introduction}

The design of some effective real-time monitoring architectures of widearea power systems with the main goal of providing some reliable information

\footnotetext{
${ }^{1}$ A preliminary version of this paper was presented at IFAC PPPSC 2012, in Toulouse, France.
}

Preprint submitted to CEP - Special Issue on Power System Control December 2, 2013 
on the health of the system, remains a big challenge, especially in the context of large transient phenomena. Nowadays communication networks plays a key role for providing reliable measurement data with minimum latency in the context of wide-area power systems. Wireless communications (both WIMAX and $3 \mathrm{G} / 4 \mathrm{G}$ ) are expected to wide-area data network coverage (see Akyol (2010)), especially in remote regions where conventional public communication networks are not available. Traditional monitoring of power grids is ensured by Remote Terminal Units (RTU), which provide real/reactive power flows, real/reactive power injections and voltage magnitude measurements. The introduction of PMUs by Phadke (1983) is a big step forward since it provided some additional measurements such as voltage and current phasor measurements. PMUs can provide very accurate data since they are synchronized from the common global positioning system (GPS) radio clock. However it is well recognized that the use of PMUs is more demanding in terms of data flow rates than traditional RTUs (see Narendra (2008)).

On the one hand, optimal PMU location is not a new topic since some previous works have been dedicated to this issue (see Baldwin (1993), Gou (2008), Xu (2004), for instance.) Hovever to the best of my knowledge, the communication infrastructure costs are never been taken into account in the design. On the other hand, dynamical state observer design capable of accurately estimating complex nonlinear transients remains a rather widely open issue (see Huang (2007) for a Kalman Filter application), since most of the available approaches in the litterature are based on steady-state estimation techniques.

On the basis of these statements, several issues will be considered in this paper:

- First, in order to effectively track the complex transient behavior of power systems, dynamical observability, rather than conventional static observability, will be considered.

- Second, the optimal location of some PMUs and PDCs will be considered. Attention will be paid to the issue of providing engineers with monitoring infrastructure design tools. The monitoring infrastructure must be understood as the choice of the power system state variables to be measured (through the optimal location of PMUs) by maximizing an observability index, together with the optimization of the communication network which will be used for both the collection and the 
transmission of sensor measurements. The communication network optimization will be viewed as the problem of optimally locating some PDCs at the vicinity of grid buses, by minimizing the induced communication costs. A budgetary constraint involving the PMU-PDC acquisition costs will be also considered.

- Third, the design of a nonlinear state observer taking advantage of the here-proposed monitoring infrastructure will be considered.

This paper extends the optimal sensor location approach proposed in Nguyen (2008) by including communication network design. Furthermore, Extended Kalman Filtering is used to design a dynamical state observer for wide-area power systems described by algebraic-differential equations, without needing nonlinear inversion techniques. This paper also extends the work proposed in Georges (2012), by assuming that the number of both PMUs and PDCs is not fixed and considering a more complex application, i.e., the IEEE 10 generator 39 bus New England power system. The application of simulated annealing to solve the NP-hard combinatorial optimal location problem is also considered in this paper.

The paper is now organized as follows: Section 2 is dedicated to some background on wide-area power system modeling.Section 3 is dedicated to a discussion on observability measurement tools based on observability gramians. In section 4, the new optimal design methodology for wide-area monitoring is proposed based on observability gramian. Section 5 describes an EKF-based dynamical state observer. Section 6 is devoted to application of the methodology to the IEEE 39-bus test system. Finally some conclusions and perspectives are provided.

\section{Nomenclature}

Subscript $i$ denotes the ith generator.

$\delta_{i} \quad$ Angle of the ith generator in radian.

$\theta_{i} \quad$ Bus $i$ phase angle, in radian. 


$\begin{array}{cc}\omega_{i} & \text { Relative speed in rad/s w.r.t. synchronous speed } \omega_{0} . \\ \omega_{0} & \text { Synchronous speed in rad/s }\left(\omega_{0}=2 \pi 60, \text { in a } 60 H z \text { network). }\right. \\ P_{i}^{m} & \text { Mechanical input power, in p.u.. } \\ P_{G i} & \text { Active power delivered, in p.u.. } \\ Q_{G i} & \text { Reactive power, in p.u.. } \\ E_{q i}^{\prime} & \text { Transient EMF in quadrature axis, in p.u.. } \\ \omega_{0} & \text { Synchronous machine spedd, in rad/s. } \\ D_{i} & \text { Per unit damping constant. } \\ H_{i} & \text { Inertia constant in second. } \\ T_{d 0 i}^{\prime} & \text { Direct axis transient short circuit time constant, in s. } \\ x_{d i} & \text { Direct axis reactance, in p.u.. } \\ x_{d i}^{\prime} & \text { Direct axis transient reactance, in p.u.. } \\ K_{a i} & \text { Exciter gain, in p.u.. } \\ T_{a i} & \text { Exciter voltage in p.u.. } \\ E_{f d i} & \text { Exciter voltage setpoint in p.u.. } \\ E_{f d 0 i} & \text { Susceptance of admittance matrix element } i, j . \\ B_{i j} & \text { Conductance of admittance matrix element } i, j . \\ G_{i j} & \text { Load active power at bus } i, \text { in p.u.. } \\ P_{L i} & \text { Load reactive power at bus } i, \text { in p.u.. } \\ Q_{L i} & \text { Voltage at bus } i, \text { in p.u.. } \\ V_{i} & \text { Voltage setpoint at bus } i, \text { in p.u.. } \\ V_{r e f i} & \text { Set of bus indices connected to bus } i, \text { including } i . \\ C_{i} & \end{array}$

\section{Power System Modeling for Wide-Area Monitoring}

A multi-machine power system, with $N$ generators connected to a grid made of $M$ buses, where $M \geq N$, is considered in this paper. Using a oneaxis model of each generator ${ }^{2}$, the model of the $i$ th generator equiped with a dynamical exciter can be expressed as follows (see Ilic (2000) for example):

\section{Mechanical Dynamics of Generator $i$ :}

$$
\dot{\delta}_{i}=\omega_{i}
$$

\footnotetext{
${ }^{2}$ For sake of simplicity and without any restriction, a two-axis or a more detailed model of the generators is not considered here.
} 


$$
\dot{\omega}_{i}=-\frac{D_{i}}{2 H_{i}} \omega_{i}+\frac{\omega_{0}}{2 H_{i}}\left(P_{i}^{m}-P_{G i}\right)
$$

Electrical Dynamics of Generator $i$ :

$$
\begin{aligned}
\dot{E}_{q i}^{\prime} & =\frac{1}{T_{d 0 i}^{\prime}}\left(E_{f d i}-\frac{x_{d i}}{x_{d i}^{\prime}} E_{q i}^{\prime}-\frac{\left(x_{d i}-x_{d i}^{\prime}\right)}{x_{d i}^{\prime}} V_{i} \cos \left(\delta_{i}-\theta_{i}\right),\right. \\
\dot{E}_{f d i} & =-\frac{1}{T_{a i}}\left(E_{f d i}-E_{f d 0 i}\right)+\frac{K_{a i}}{T_{a i}}\left(V_{r e f i}-V_{i}\right) .
\end{aligned}
$$

Electrical Equations at Generator bus $i, i=1, \ldots, N$ :

$$
\begin{aligned}
P_{G i} & =\frac{E_{q i}^{\prime} V_{i}}{x_{d i}^{\prime}} \sin \left(\delta_{i}-\theta_{i}\right) \\
Q_{G i} & =\left(E_{q i}^{\prime} V_{i} \cos \left(\delta_{i}-\theta_{i}\right)-V_{i}^{2}\right) / x_{d i}^{\prime} \\
P_{G i} & =\sum_{k \in C_{i}} V_{i} V_{k}\left(G_{i k} \cos \theta_{i k}+B_{i k} \sin \theta_{i k}\right)+P_{L i} \\
Q_{G i} & =\sum_{k \in C_{i}} V_{i} V_{k}\left(G_{i k} \sin \theta_{i k}-B_{i k} \cos \theta_{i k}\right)+Q_{L i}
\end{aligned}
$$

where $\theta_{i k}=\theta_{i}-\theta_{k}$.

Electrical Equations at the Non Generator Bus $j, j=1, \ldots, M-N$ :

$$
\begin{aligned}
P_{L j}+\sum_{k \in C_{j}} V_{j} V_{k}\left(G_{j k} \cos \theta_{j k}+B_{j k} \sin \theta_{j k}\right) & =0 \\
Q_{L j}+\sum_{k \in C_{j}} V_{j} V_{k}\left(G_{j k} \sin \theta_{j k}-B_{j k} \cos \theta_{i k}\right) & =0 .
\end{aligned}
$$

The overall dynamical model of the power system may be expressed as the following algebraic-differential system:

$$
\begin{aligned}
\dot{x} & =F(x, z, u), \\
0 & =G(x, z, w),
\end{aligned}
$$

where $x$ denotes the vector of the $N$ generator state variables $\left(\delta_{i}, \omega_{i}, E_{q i}^{\prime}, E_{f d i}\right)$, $i=1, \ldots, N$ and possibly the state variables of additional controllers such as PSS or FACTS, $z$ is the $2 M$ vector of the voltage magnitude and phase angle 
at the $M$ buses. $u$ is the vector of reference control inputs, i.e. the mechanical power and the reference inputs of each controllers. $w$ is the vector of load currents at each bus. $w$ can also include interconnection variables if the studied system is part of a larger power system. $F$ denotes the vector field of the differential part of the state-space representation, while $G$ is a nonlinear function with the same dimensions as the ones of vector $z$. If additional controllers (PSS, FACTS, AVR) are introduced, system model (11)-(12) can be extended as follows:

$$
\begin{aligned}
\dot{x} & =F(x, z, u), \\
\dot{c} & =I(c, x, z, v), \\
0 & =G(x, z, w), \\
u & =\operatorname{sat}(\phi(c))
\end{aligned}
$$

where $I$ denotes the vector field of the controllers (PSS, AVR, FACTS controllers), $c$ is the controller state vector, and $v$ is a vector of controller setpoints. $x$ may also include additional states of FACTS devices. stat denotes a saturation function defined as follows:

$$
\operatorname{sat}(u)=\left\{\begin{array}{c}
\underline{u}, \text { if } u<\underline{u} \\
u, \text { if } \underline{u} \leq u \leq \bar{u} \\
\bar{u}, \text { if } u>\bar{u}
\end{array}\right.
$$

If controller state $c$ is known, an extended-Kalman observer design can be performed based on the reduced system defined by states $x$ and $z$, under the assumption that both $F$ and $G$ are differentiable and a local detectability property holds.

For the goal of both simulation and state-observer design based on time integration techniques, it is convenient to use a differential version of the algebraic equations by introducing the time-derivative of $G$ :

$$
\dot{G}(x, z, w)=\frac{\partial G}{\partial x} \dot{x}+\frac{\partial G}{\partial z} \dot{z}+\frac{\partial G}{\partial w} \dot{w} .
$$

and the condition $\dot{G}=0$. However it is more appropriate to ensure numerical stability by introducing a stabilizing term as follows:

$$
\dot{G}(x, z, w)=-\frac{1}{\epsilon} G(x, z, w),
$$


with $\epsilon>0$. This equation is equivalent to

$$
\dot{z}=-\frac{\partial G^{-1}}{\partial z}\left[\frac{\partial G}{\partial x} F(x, z, u)+\frac{\partial G}{\partial w} \dot{w}+\frac{1}{\epsilon} G(x, z, w)\right],
$$

provided that the Jacobian matrix $\frac{\partial G}{\partial z}$ has full rank (except on a subset of null measure corresponding to some singular configurations).

\section{Some remarks:}

1. The classical approach consists in using the implicit function theorem, in order to locally express $z$ as a function $\Psi(x, w)$ provided that the Jacobian matrix $\frac{\partial G}{\partial z}$ has full rank. In most of the cases, it is not possible to get an explicit expression of $\Psi(x, w)$ and some NewtonRaphson-like methods are thus needed to compute $z$. The here-proposed differential version avoids such iterative computations of the implicit solution, except for the initial state.

2. Equation (19) may be interpreted as a singularly-perturbated version of $G(x, z, w)=0$, since it may be rewritten as $\epsilon \dot{G}(x, z, w)=-G(x, z, w)$. In the case of long interconnection lines, it is well known that a static model of the form $G(x, z, w)=0$ may be not suitable, since travelling waves may occur. $\epsilon \dot{G}(x, z, w)=-G(x, z, w)$ may also be viewed as a way of integrating such transient phenomena.

3. It can be easily shown that the solution of (19) is given by

$$
G(x(t), z(t), w(t))=e^{-\frac{t}{\epsilon}} G(x(0), z(0), w(0)) .
$$

This means that the trajectory of $x$ and $z$ are constrained to remain on the manifold defined by $G(x, z, w)=0$, provided that $w$ and its time derivative $\dot{w}$ are bounded, when initial states $x(0)$ and $z(0)$ are such that $G(x(0), z(0), w(0))=0$. When the initial states do not belong to the manifold, eqn (16) ensures exponential attractivity to the manifold.

4. This also means that any linearized dynamics around a given equilibrium state exhibits $2 M$ eigenvalues equal to $-\frac{1}{\epsilon}$ (in order to prove this assertion, consider the change of coordinates $z \rightarrow G(x, z, w))$.

In addition to the state-representation (11)-(12), measurement vector $y$ needs to be defined:

$$
y=H(x, z)
$$


where $H(x, z)$ defines the measured outputs as a function of the states $x$ and $z . H$ is supposed to be at least continuously differentiable. When some traditional sensors are used to measure active and reactive powers, the related measured variables will be expressed as a nonlinear function of the state. When some sensors (PMUs for example) are used to measure phase angle and voltage magnitudes, the related component of $y$ will be simply one of the state components. Again, if some current measurements are performed, the related output will be a nonlinear function of the states.

Finally, a nonlinear fully-differential state representation of the power system is given by

$$
\begin{aligned}
\dot{x} & =F(x, z, u), \\
\dot{z} & =-\frac{\partial G^{-1}}{\partial z}\left[\frac{\partial G}{\partial x} F(x, z, u)+\frac{\partial G}{\partial w} \dot{w}\right. \\
& \left.+\frac{1}{\epsilon} G(x, z, w)\right], \\
y & =H(x, z) .
\end{aligned}
$$

In what follows, it is assumed that the vector of load and interconnection currents $w$ are known via measurements, together with their time-derivatives (through some appropriate filters for example) $)^{3}$. This new state representation will be used to derive a dynamical state observer in section 5 .

\section{Observability Analysis based on the Observability Gramian}

For asymptotically stable linear systems (i.e. the spectrum of matrix $A$ belongs to the left part of the complex plane) defined by

$$
\begin{aligned}
& \dot{x}=A x+B u, \\
& y=C x+D u,
\end{aligned}
$$

a measure of observability is given by the output energy function generated by any intial state $x_{0}$ (when $u=0$ ) and given by

$$
E_{o}\left(x_{0}\right)=\int_{0}^{\infty}\|y(t)\|^{2} d t=x_{0}^{T}\left[\int_{0}^{\infty} e^{A^{T} t} C^{T} C e^{A t} d t\right] x_{0} .
$$

\footnotetext{
${ }^{3}$ An extension to the case of some non measured $w$ is possible, provided that the system observability condition is satisfied, by introducing an extended version of (23)-(25) obtained by adding the dynamics $\dot{w}=0$ in the observer design.
} 
The matrix $W_{o}=\int_{0}^{\infty} e^{A^{T} t} C^{T} C e^{A t} d t$ is called observability Gramian of the system and is given as solution of the following Lyapunov equation (Moore (1981))

$$
A^{T} W_{o}+W_{o} A+C^{T} C=0 .
$$

It is well known that the nullspace of $W_{o}$ coincides with the unobservable subspace of the system, i.e, when the pair $(C, A)$ is $\operatorname{detectable}^{4}, W_{o}$ is a nonnegative definite matrix.

In Zhou (1999), the notion of Gramian has been extended to the case of unstable systems (without any poles on the imaginary axis), by considering the solutions $Y, W_{o}$ of the following algebraic matrix equations:

$$
\begin{gathered}
A Y+Y A^{T}-Y C^{T} C Y=0, \\
A_{o}^{T} W_{o}+W_{o} A_{o}+C^{T} C=0,
\end{gathered}
$$

where $A_{o}=A-Y C^{T} C$ and with $W_{o}=W_{o}^{T} \geq 0$ and $Y=Y^{T} \geq 0$.

The first (Bernoulli) equation ensures the projection of the unstable eigenvalues of $A$ to the left part of the complex plane, symmetrically with respect to the imaginary axis (by using an "implicit" Kalman filter). When the system is stable, we use in fact the trivial solution $Y=0$. As a consequence $W_{o}$ may be used as a measure of the "level of observability" induced by a particular choice or location of sensors in the system. An optimal location of sensors will consist for instance in maximizing the minimum singular value of $W_{o}$ (see Georges (1995)) or the trace of $W_{o}$, which represents the sum of the singular values of $W_{o}$ (see Georges (2011)). Some efficient numerical methods were proposed to compute large-scale Lyapunov-type equations. For instance reference (Jaimoukha (1994)) provides a computational approach based on Krylov subspaces. This is precisely the approach that will be used for designing an optimal PMU-based monitoring architecture.

For that purpose, the local linearization of the system (11)-(12) around any equilibrium set point (denoted by the subscript "e") is introduced:

$$
\dot{\tilde{x}}=A_{e}^{1} \tilde{x}+A_{e}^{2} \tilde{z}+B_{e}^{1} \tilde{u},
$$

\footnotetext{
${ }^{4} \mathrm{~A}$ system is said to be detectable, if and only if the non observable states of the system are asymptotically stable.
} 


$$
\begin{aligned}
0 & =A_{e}^{3} \tilde{x}+A_{e}^{4} \tilde{z}+B_{e}^{2} \tilde{w} \\
\tilde{y} & =C_{e}^{1} \tilde{x}+C_{e}^{2} \tilde{z}
\end{aligned}
$$

where $A_{e}^{1}, A_{e}^{2}$ and $B_{e}^{1}$ are the Jacobian matrices of $F$ with respect to $x, z$ and $u$ respectively, while $A_{e}^{3}, A_{e}^{4}$, and $B_{e}^{2}$ are the Jacobian matrices of $G$ with respect to $x, z$, and $w$ respectively, and finally, $C_{e}^{1}$ and $C_{e}^{2}$ are the Jacobian matrices of $H$ with respect to $x$ and $z$, evaluated at the equilibrium point. : denotes the discrepancy between the variable and its equilibrium value.

By using that $A_{e}^{4}$ is invertible, $\tilde{z}$ can be expressed as $\tilde{z}=-A_{e}^{4-1}\left[A_{e}^{3} \tilde{x}+\right.$ $\left.B_{e}^{2} \tilde{w}\right]$. Consequently, the algebraic part of the model and the state $\tilde{z}$ can be eliminated:

$$
\begin{aligned}
& \dot{\tilde{x}}=\left(A_{e}^{1}-A_{e}^{2} A_{e}^{4^{-1}} A_{e}^{3}\right) \tilde{x}+B_{e}^{1} \tilde{u}-A_{e}^{2} A_{e}^{4^{-1}} B_{e}^{2} \tilde{w}, \\
& y=\left(C_{e}^{1}-C_{e}^{2} A_{e}^{4^{-1}} A_{e}^{3}\right) \tilde{x}-C_{e}^{2} A_{e}^{4^{-1}} B_{e}^{2} \tilde{w}
\end{aligned}
$$

The observability Gramian of the power system is then computed as solution of (30) and (31), where

$$
\begin{array}{r}
\left(A_{e}^{1}-A_{e}^{2} A_{e}^{4^{-1}} A_{e}^{3}\right) Y+Y\left(\left(A_{e}^{1}-A_{e}^{2} A_{e}^{4^{-1}} A_{e}^{3}\right)^{T}\right. \\
-Y\left(C_{e}^{1}-C_{e}^{2} A_{e}^{4^{-1}} A_{e}^{3}\right)^{T}\left(C_{e}^{1}-C_{e}^{2} A_{e}^{4^{-1}} A_{e}^{3}\right) Y=0 \\
A_{o}^{T} W_{o}+W_{o} A_{o} \\
+\left(C_{e}^{1}-C_{e}^{2} A_{e}^{4^{-1}} A_{e}^{3}\right)^{T}\left(C_{e}^{1}-C_{e}^{2} A_{e}^{4-1} A_{e}^{3}\right)=0,
\end{array}
$$

where

$$
A_{o}=\left(A_{e}^{1}-A_{e}^{2} A_{e}^{4^{-1}} A_{e}^{3}\right)-Y\left(C_{e}^{1}-C_{e}^{2} A_{e}^{4^{-1}} A_{e}^{3}\right)^{T} .
$$

In what follows, $A_{e}^{1}-A_{e}^{2} A_{e}^{4^{-1}} A_{e}^{3}$ and $C_{e}^{1}-C_{e}^{2} A_{e}^{4^{-1}} A_{e}^{3}$ will be denoted as $A$ and $C$ respectively ${ }^{5}$.

\section{Design of a Monitoring Architecture Based on the Optimal Lo- cation of PMUs and PDCs}

The problem consists in adding $N_{p m u}$ PMUs and $N_{p d c}$ PDCs to an existing monitoring infrastructure equiped with some traditional sensors or not, where $N_{p m u}$ and $N_{p d c}$ have to be minimized.

\footnotetext{
${ }^{5}$ In order to ensure existence of solutions to (37) and (38), dynamics of the reference generator has to be eliminated.
} 
Each of the $N_{p m u}$ PMUs is supposed to be connected to one and only one of the $N_{p d c}$ PDCs, which are themselves connected to a SCADA master station, according to Fig. 1.

The main goal is now to derive an optimal monitoring architecture by taking the following objectives into account:

1. Both the PMUs and the PDCs must be located at some well chosen system buses. The optimization should be performed on the basis of a large number of system set point scenarios.

2. The optimal location of both the PMUs and the PDCs must be ensured to account the objectives of maximizing observability while reducing the communication infrastructure induced costs (depending on the used technology, for instance, WIMAX, coaxial, or optical communications). The communication infrastructure costs are assumed to depend on the length of the communication link and possibly the geographical location of the devices in order to take some civil engineering constraints into account.

These objectives can be fulfilled by solving the following integer programming problem (denoted OLP):

$$
\begin{array}{r}
\left.\max _{S_{i j}, \alpha_{i j}, \beta_{i j} \in\{0,1\}} \operatorname{Erace}\left(W_{o}(S, \omega)\right)\right] \\
-\sum_{i=1}^{\bar{N}_{p m u}} \sum_{j=1}^{\bar{N}_{p d c}} \alpha_{i j} \max \left(0, I_{p d c_{j}}^{b}\right) \times \max \left(0, I_{p m u_{i}}^{b}\right) \times C_{c}\left(I_{p m u_{i}}^{b}, I_{p d c_{j}}^{b}\right)
\end{array}
$$

subject to

$$
\begin{array}{r}
\sum_{j=1}^{M} S_{i j} \leq 1, i=1, \ldots, \bar{N}_{p m u}, \\
\sum_{i=1}^{\bar{N}_{p m u}} S_{i j} \leq 1, j=1, \ldots, M, \\
\left(\sum_{k=1}^{M} S_{i k}-1\right)\left(\sum_{j=1}^{\bar{N}_{p d c}} \alpha_{i j}\right)=0, i=1, \ldots, \bar{N}_{p m u}, \\
\left(\sum_{k=1}^{M} S_{i k}\right)\left(\sum_{j=1}^{\bar{N}_{p d c}} \alpha_{i j}-1\right)=0, i=1, \ldots, \bar{N}_{p m u},
\end{array}
$$


$\Delta$



0xy

Figure 1: Example of the location of 5 PMUs connected to 2 PDCs. 


$$
\begin{array}{r}
\sum_{j=1}^{M} \beta_{i j} \leq 1, i=1, \ldots, \bar{N}_{p d c}, \\
\sum_{i=1}^{\bar{N}_{p m u}} \beta_{i j} \leq 1, j=1, \ldots, M \\
N_{p m u} \times C_{p m u}^{a}+\sum_{i=1}^{N_{p d c}} C_{p d c}^{a}\left(N_{p d c_{i}}^{c}\right) \leq \bar{C}_{a}, \\
\sum_{i=1}^{\bar{N}_{p m u}} \alpha_{i j} \leq \bar{N}_{c}, j=1, \ldots, \bar{N}_{p d c}, \\
\sum_{i=1}^{\bar{N}_{p d c}} \sum_{j=1}^{M} \beta_{i j} \geq \underline{N}_{p d c},
\end{array}
$$

where $S_{i j}$ is equal to 1 (otherwise 0 ), when the $i$ th PMU is located at bus $j$; $\alpha_{i j}$ is equal to 1 (otherwise 0 ), when the $i$ th PMU is connected to the $j$ th PDC; $\beta_{i j}$ is equal to 1 (otherwise 0 ), when the $i$ th PDC is located at bus j. $\bar{N}_{p m u}$ (resp. $\bar{N}_{p d c}$ ) is the maximum number of PMUs (resp. of PDCs); $I_{p m u_{i}}^{b}$ (resp. $I_{p d c_{i}}^{b}$ ) is the bus index of the $i$ th PMU (resp. the bus index of the $i$ th PDC). $I_{p m u_{i}}^{b}\left(\operatorname{resp} . I_{p d c_{i}}^{b}\right)$ is equal to 0 , when $\sum_{j=1}^{M} S_{i j}=0$ (resp. $\left.\sum_{j=1}^{M} \beta_{i j}=0\right)$. Number of PMUs $N_{p m u}$ (resp. number of PDCs $N_{p d c}$ ) is given by $N_{p m u}=\sum_{i=1}^{\bar{N}_{p m u}} \sum_{j=1}^{M} S_{i j}\left(\right.$ resp. $\left.N_{p d c}=\sum_{i=1}^{\bar{N}_{p d c}} \sum_{j=1}^{M} \beta_{i j}\right)$. Number of PMUs connected to PDC $i$ is given by $N_{p d c_{i}}^{c}=\sum_{j=1}^{\bar{N}_{p m u}} \alpha_{j i} . \bar{N}_{c}$ is the maximum number of PMU connexions per PDC allowed. $\underline{N}_{p d c}$ is the minimum number of PDC. $C_{p m u}^{a}$ is the unitary PMU acquisition cost. $C_{p d c}^{a}$ is the unitary PDC acquisition cost which may be a function of the number of PMUs. $\bar{C}_{a}$ is the maximum PMU-PDC acquisition cost. $B_{p d c_{i}}^{c}$ connected to PMU $i, B_{p d c_{i}}^{c}=\sum_{j=1}^{\bar{N}_{p m u}} \alpha_{j i} . C_{c}$ is the unitary communication infrastructure cost of any link connecting a 
PMU to a PDC.

\section{Discussions on the cost functionals:}

- $E[$.$] is the expected value defined on a probability space defined by$ a large number $N_{s c}$ of scenarios $\omega_{i}$ corresponding each to one (non necessarily stable) equilibrium set point of the power system. Each scenario has its own occurence probability $P\left(\omega_{i}\right)$. Consequently,

$$
E\left[\operatorname{trace}\left(W_{o}(S, \omega)\right)\right]=\sum_{i=1}^{N_{s c}} \operatorname{trace}\left(W_{o}\left(S, \omega_{i}\right)\right) P\left(\omega_{i}\right) .
$$

Each $W_{o}\left(S, \omega_{i}\right)$ is solution of (30) and (31):

$$
\begin{array}{r}
A\left(\omega_{i}\right) Y\left(S, \omega_{i}\right)+Y\left(S, \omega_{i}\right) A^{T}\left(\omega_{i}\right) \\
-Y\left(S, \omega_{i}\right) C^{T}\left(S, \omega_{i}\right) C\left(S, \omega_{i}\right) Y\left(S, \omega_{i}\right)=0 \\
A_{o}^{T}\left(\omega_{i}\right) W_{o}\left(S, \omega_{i}\right)+W_{o}\left(S, \omega_{i}\right) A_{o}\left(\omega_{i}\right) \\
+C^{T}\left(S, \omega_{i}\right) C\left(S, \omega_{i}\right)=0
\end{array}
$$

where $A_{o}=A-Y C^{T} C$.

- $C_{c}\left(I_{p m u_{i}}^{b}, I_{p d c_{j}}^{b}\right)$ is the communication infrastructure cost induced by the location of both the $i$ th PMU and the $j$ th PDC. $C_{c}$ is defined as an increasing function of the length of the link connecting the $i$ th PMU to the $k$ th PDC. $C_{c}$ may also include some cost components related to the locations of the considered PMU and PDC by themselves: For instance, the erection cost of a communication tower in the presence of environmental obstacles.

\section{Discussions on the constraints:}

- Constraints (40) are introduced to ensure that each of the $N_{p m u}$ PMUs is connected to only one bus.

- Constraints (41) are introduced to avoid that each PMU is connected to more than one PMU.

- Constraints (42) ensure that if PMU $i$ is not connected to a bus, then $\alpha_{i j}, i=1, \ldots, \bar{N}_{p d c}$ (no connexion to a PDC). 
- Constraints (43) ensure that if PMU $i$ is connected to a bus, then PMU $i$ is also connected to a PDC.

- Constraints (44) are introduced to ensure that each PDC is connected to only one bus.

- Constraints (45) are introduced to avoid that each of the $N_{p d c}$ PDCs is connected to more than one PMU.

- Constraint (46) is introduced to bound the upper PMU-PDC acquisition cost.

- Constraints (47) are introduced to bound the number of possible PMU connexions to a given PDC with $\bar{N}_{c}$.

- Constraint (48) is introduced to ensure existence of at least $\underline{N}_{p d c}$ PDC.

\subsection{Numerical computation based on simulated annealing}

This large-scale highly combinatorial (NP-hard) optimization problem $\left(\right.$ with $\bar{N}_{p m u} \times M+\bar{N}_{p d c} \times \bar{N}_{p m u}+\bar{N}_{p d c} \times M$ decision variables and $3 \bar{N}_{p m u}+$ $2 \bar{N}_{p d c}+2 M+2$ constraints) cannot be solved by an enumerative method and an optimal solution should be computed by using any available integer programming methods (such as Branch and Bound methods, simulated annealing, genetic algorithms ...), (see Gou (2008) for example). The combinatorial complexity depends on the number of system buses. However the computation time of the observability index depends on the number of generator and grid states, but it does not directly affect the combinatorial complexity. It can be easily shown also that the average computation time linearly depends on the number of set point scenarios used to define the observability index.

In order to illustrate the combinatorial complexity of the problem, let $T_{c}$ denote the CPU time needed to compute the optimal solution a of a rather simple problem of locating $k$ PMUs on a $n$-bus power system with a single PDC connecting the PMUs to the SCADA, which is given by the following formula:

$$
T_{c}=\frac{n !}{k ! \times(n-k) !} \times t_{c p u}^{c o s t} \times N_{s c}
$$

where $t_{c p u}^{c o s t}$ is the unitary CPU time needed for evaluating the cost function related to a given configuration of the PMUs for a given set point scenario. 
For instance, if $n=39, k=20, t_{c p u}^{c o s t}=1 \mathrm{~ms}$, and $N_{s c}=100$, the timed need is greater than 218 years!

In this paper, the computation of a numerical solution is performed by using simulated annealing (see Kirkpatrick (1983)).

Simulated annealing is an iterative method primarily intended to solving unconstrained optimization problems. The method mimics the physical process of heating a material and then slowly cooling it in order to eliminate some material defects by reaching an equilibrium state corresponding to a minimum of the material energy.

At each temperature iteration of the simulated annealing algorithm, a new solution is randomly generated. The distance of the new solution from the current one, is based on a probability distribution proportional to the temperature. The algorithm accepts all new solutions that lower the cost function, but also, with a certain probability, solutions that increase the cost function. By generating solutions that increase the cost function, the algorithm avoids being trapped in local minima and thus has been proved to provide better minimal solutions. It has been also proved (see Granville (1994)) that simulated annealing is able to provide an optimal solution to any unconstrained optimization problem provided that the number of temperature iterations tends to infinity.

Integer programming problem OLP is a constrained optimization problem which can be solved by simulated annealing thanks to a exterior penalty method. This method transforms the problem into an unconstrained optimization problem (OLPP), by adding penalty functions to the initial cost function. The new unsconstrained problem is described as follows:

$$
\begin{array}{r}
\max _{S_{i j}, \alpha_{i j}, \beta_{i j} \in\{0,1\}} E\left[\operatorname{trace}\left(W_{o}(S, \omega)\right)\right] \\
-\sum_{i=1}^{\bar{N}_{p m u}} \sum_{j=1}^{\bar{N}_{p d c}} \alpha_{i j} \max \left(0, I_{p d c_{j}}^{b}\right) \times \max \left(0, I_{p m u_{i}}^{b}\right) \times C_{c}\left(I_{p m u_{i}}^{b}, I_{p d c_{j}}^{b}\right) \\
+K_{p} \sum_{i=1}^{\bar{N}_{p m u}} \max \left(0, \sum_{j=1}^{M} S_{i j}-1\right)^{2}+K_{p} \sum_{j=1}^{M} \max \left(0, \sum_{i=1}^{\bar{N}_{p m u}} S_{i j}-1\right)^{2} \\
+K_{p} \sum_{i=1}^{\bar{N}_{p m u}}\left(\left(\sum_{k=1}^{M} S_{i k}-1\right)\left(\sum_{j=1}^{\bar{N}_{p d c}} \alpha_{i j}\right)\right)^{2}+K_{p} \sum_{i=1}^{\bar{N}_{p m u}}\left(\left(\sum_{k=1}^{M} S_{i k}\right)\left(\sum_{j=1}^{\bar{N}_{p d c}} \alpha_{i j}-1\right)\right)^{2}
\end{array}
$$




$$
\begin{array}{r}
+K_{p} \sum_{i=1}^{\bar{N}_{p d c}} \max \left(0, \sum_{j=1}^{M} \beta_{i j}-1\right)^{2}+K_{p} \sum_{j=1}^{M} \max \left(0, \sum_{i=1}^{\bar{N}_{p m u}} \beta_{i j}-1\right)^{2} \\
+K_{p} \max \left(0, N_{p m u} \times C_{p m u}^{a}+\sum_{i=1}^{N_{p d c}} C_{p d c}^{a}\left(N_{p d c_{i}}^{c}\right)-\bar{C}_{a}\right)^{2} \\
+K_{p} \sum_{j=1}^{\bar{N}_{p d c}} \max \left(0, \sum_{i=1}^{\bar{N}_{p m u}} \alpha_{i j}-\bar{N}_{c}\right)^{2}+K_{p} \max \left(0, \underline{N}_{p d c}-\sum_{i=1}^{\bar{N}_{p d c}} \sum_{j=1}^{M} \beta_{i j}\right)^{2}
\end{array}
$$

where $K_{p}$ is a penalty coefficient, which has to be chosen small, but large enough to ensure the constraints are satisfied.

\section{Dynamical State-Observer Design}

The here-proposed derivation of a centralized state observer for wide-area monitoring is based on both the nonlinear differential model (23)-(25) and the use of an Extended Kalman Filter, whose gain is computed apart from the linearized dynamics of the reduced system (35)-(36) around the estimated state trajectory (see Gibbs (2011) for example):

$$
\begin{aligned}
\dot{\hat{x}} & =F(\hat{x}, \hat{z}, u)+L(\hat{x}, \hat{z}, u, w)(y-H(\hat{x}, \hat{z})), \\
\dot{\hat{z}} & =-\frac{\partial G^{-1}}{\partial z}\left[\frac{\partial G}{\partial x} F(\hat{x}, \hat{z}, u)+\frac{\partial G}{\partial w} \hat{\dot{w}}+\frac{1}{\epsilon} G(\hat{x}, \hat{z}, w)\right], \\
\hat{\dot{w}} & =k_{c}(w-\hat{w}), k_{c}>0
\end{aligned}
$$

where gain matrix $L$ is obtained from the solution $P(t)$ of the following differential Riccati equation:

$$
\begin{aligned}
\dot{P}(t) & =P(t) A(\hat{x}(t), \hat{z}(t), u(t), w(t))^{T}+A(\hat{x}(t), \hat{z}(t), u(t), w(t)) P(t) \\
& -P(t) C_{a}(\hat{x}, \hat{z})^{T} R^{-1} C_{a}(\hat{x}, \hat{z}) P(t)+Q, \\
P(0) & =Q_{0},
\end{aligned}
$$

where $A$ and $C$ are the already-described matrices of the reduced linearized system (35)-(36), obtained from the Jacobian matrices of the linearized dynamics of (11)-(22) evaluated around the estimated trajectory $(\hat{x}(t), \hat{z}(t))$, $u(t)$, and $w(t)$. Matrices $Q_{0}, Q$ and $R$ are chosen in order to represent the covariance matrix of the initial state, of the state equation disturbance and of the measurement disturbance, respectively. 
Gain matrix $L$ is given by

$$
L(\hat{x}(t), \hat{z}(t), u(t), w(t))=P(t) C^{T}(\hat{x}(t), \hat{z}(t)) R^{-1} .
$$

(56) is the nonideal differentiator which provides an estimate of $\dot{w}$.

\section{Some remarks.}

1. There is no need to invert the algebraic "flow" equations (12) of the grid (for example by using a continuation method) since the observer does not contain any algebraic part.

2. Gain $L$ computation requires solving a reduced observer Riccati equation (since grid state $z$ has been eliminated). Thus the computational complexity depends only on the number of generator states.

3. Furthermore, it can also be easily shown that under the assumption that the state observer converges, the initial state estimate is not supposed to satisfy (12) to get an estimate of $x, z$ such that $(\hat{x}(t), \hat{z}(t)) \rightarrow$ $(x(t), z(t))$ of the algebraic -differential model (11)-(12), when $t \rightarrow+\infty$.

4. In pratice, the effect of various communication delays can be dealed with provided that all the phasor measurements are synchronized and the data acquisation time is the same for all the PMUs. Synchronized PMUs use the American military's Global Positioning System satellites and are capable of providing measurements with a precision of a few microseconds (see chapter 5 in Hadjsaid (2008) for instance). Synchronized PMU measurements, which correspond to a given acquisition time, can be used after data transmission, buffering and data re-ordering, in order to compute some delayed estimates of the system states, in a manner similar to video streaming.

\section{Application to the IEEE 10-generator 39-bus New England power system}

In order to illustrate both OLP and the proposed state observer behavior, the well-known IEEE New England power system benchmark was considered (see Fig. 2). All the generator, lines/transformers, and power/voltage set point data were retrieved from the website http://sys.elec.kitami-it.ac.jp-

/ueda/demo/WebPF/39-New-England.pdf. New England 39 bus system is 
a $60 \mathrm{~Hz}$ system. The network consists of 10 synchronous generators, and 46 transmission lines. Generator 2 at Bus 31 is considered as reference. A full description of the system is also available in Pai (1989).

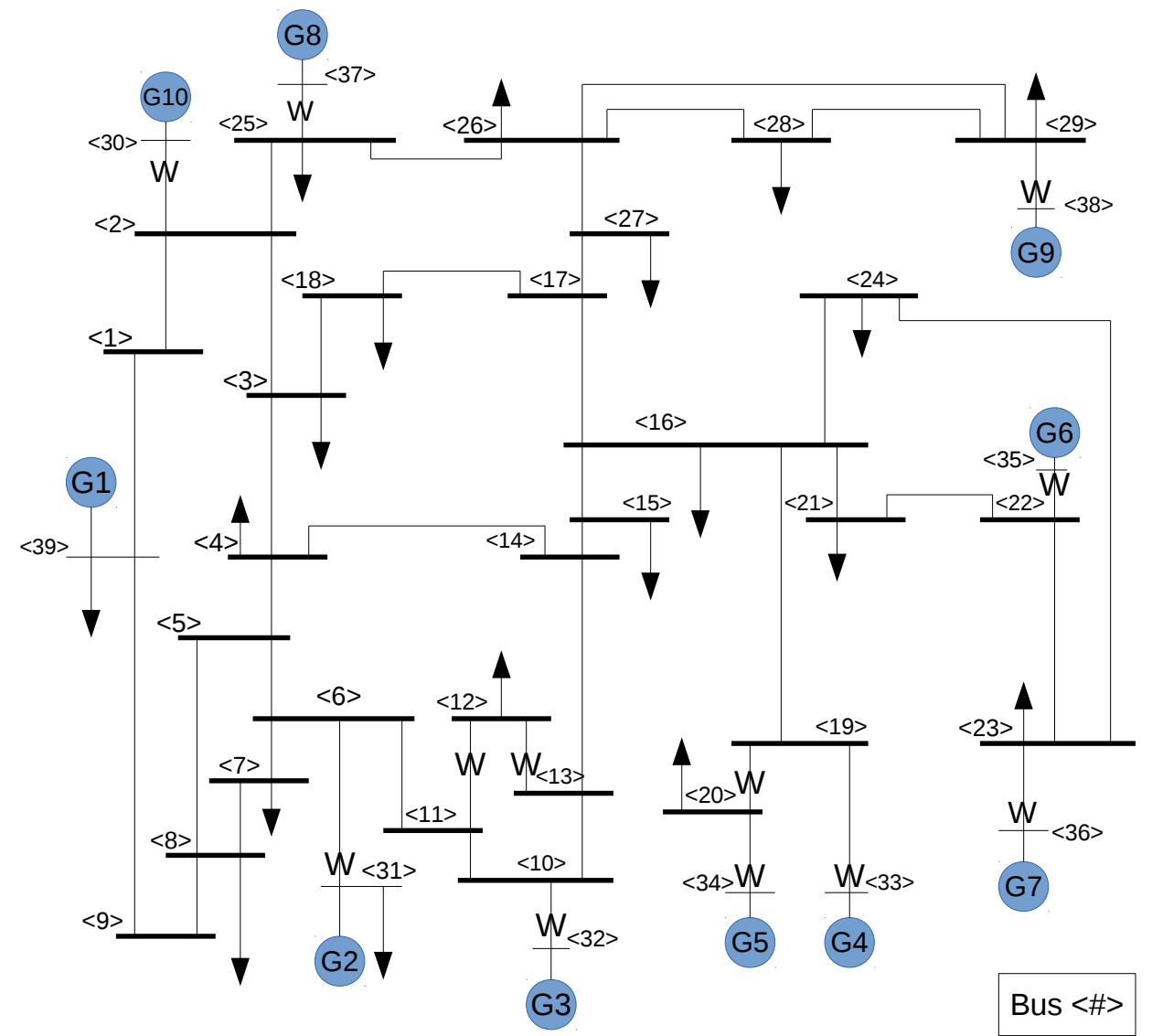

Figure 2: The IEEE 10-generator 39-bus power system.

\subsection{OLP solution}

The main goal was to compute the best location of a minimum number of PMUs dedicated to voltage phasor measurement at two of the buses, together with the optimal location of a minimum number of PDCs linked to the PMUs. 
The communication infrastructure cost was assumed to be a linear function of the communication link length between each PMU and its PDC:

$$
C_{c}\left(I_{p m u_{i}}^{b}, I_{p d c_{j}}^{b}\right)=\gamma d\left(I_{p m u_{i}}^{b}, I_{p d c_{j}}^{b}\right), i=1, \ldots, \bar{N}_{p m u}, j=1, \ldots, \bar{N}_{p d c}
$$

where $\gamma$ represents the infrastructure cost per $\mathrm{km}$ and $d\left(I_{p m u_{i}}^{b}, I_{p d c_{j}}^{b}\right)$ is the communication link length between PMU $i$ and PDC $j$. Here $\gamma$ was assumed to be equal to $1 / \mathrm{km}$ (normalized cost per $\mathrm{km}$ ).

The normalized unitary PMU-PDC acquisition costs were defined as $C_{p m u}^{a}=$ 10 , and $C_{p d c}^{a}=5$ (this unitary cost is not depending on the number of PMU connexions in this example). 20 scenarios were generated by randomly pertubating the load and the generation nominal set point by using a uniform probability distribution with standard deviation of 0.2 .

Problem OLPP was solved by using simulating annealing with penalty coefficient $K_{p}=750$ on a desktop with Intel Core i5-2320@3.00GHz and 6 Go RAM. The optim_sa function of SCILAB software ${ }^{6}$ was used to compute the simulated annealing OLPP solution.

In order to analyze the effectiveness of the simulating annealing algorithm, a comparison was made in computing the cost function value of the simulated annealing solution and the one obtained with an enumerative solution in the simple case when $\bar{N}_{p m u}=2$ and $\bar{N}_{p d c}=1$. Table 1 provides a comparison:

Table 1: Enumerative solution versus simulated annealing solution (60 iterations).

\begin{tabular}{ccc} 
Method & Cost & CPU Time \\
\hline Enumerative solution & 16788.4 & 5 hours $33 \mathrm{mns}$ \\
\hline Simulated annealing & 16781.8 & 5 hours $45 \mathrm{mns}$ \\
\hline
\end{tabular}

The relative cost discrepancy is limited to $3.9 e^{-4}$, which is a very good result. It can be noticed that, in this rather simple case, the enumerative solution is a little less CPU time consuming.

\footnotetext{
${ }^{6}$ SCILAB is an open source software for numerical computation, trademark of Scilab Entreprises SAS 2013.
} 
Then the maximum numbers of devices were given by $\bar{N}_{p m u}=25$ and $\bar{N}_{p d c}=5 . \quad \underline{N}_{p d c}=2$ and $\bar{N}_{c}=10$. Maximum acquisition budget $\bar{C}_{a}$ was fixed to 215. Tables 2, 3, and 4 sum up the results obtained with simulated annealing. In this case, it was not possible to compare simulated annealing with the enumerative method due to the hudge combinatorial explosion.

Table 2: Simulated annealing solution (60 iterations).

\begin{tabular}{cc} 
Cost & CPU Time \\
\hline 25889.7 & 6 hours $30 \mathrm{mns}$ \\
\hline
\end{tabular}

\subsection{Nonlinear state observer simulation}

The state observer described in section 5 was tested with the optimal configuration of 20 PMUs and $\epsilon=0.01$, with the data defined by Table 5 , when the power system reacts to a sudden $50 \%$ load fall at bus 15 at time instant $200 \mathrm{~ms}$, without considering frequency control mechanisms. The initial state estimates were chosen to represent $80 \%$ of the unknown states at initial equilibrium. The PMUs are used to measure voltage angles and magnitudes at buses where they are optimally located. A Gaussian measurement noise with zero mean and a standard deviation of $1 e^{-2}$ was also introduced to alter the measurements provided by each PMU. Fig. 3, 4, 5 and 6 show how well the EKF observer behaves in response to the sudden load change occuring at $t=200 \mathrm{~ms}$. On Fig. 3, the 10-generator angle estimation errors are shown to be bounded by 0.1 in magnitude, after initial transients, whose duration is limited to $150 \mathrm{~ms}$. Those transients are due to the fact the initial states are supposed to be not precisely known. The occurence of the load change at $t=200 \mathrm{~ms}$ does not introduce some new transients, that is an interesting feature of the proposed observer design. Fig. 4 corresponds to results given by Fig. 3 with a comparison between the real 10-generator angles, obtained by simulation, and the estimated ones. Fig. 5 provides a comparison between the real 10-generator relative speeds (w.r.t. $\omega_{0}$ and the estimated ones. Fig. 6 provides a comparison between the real 39-bus voltage angles and the estimated ones. Figs 5 and 6 show that the state trajectory leaves the equilibrium state after $200 \mathrm{~ms}$ : the relative speeds w.r.t. the synchronous speed of the generators increase (see Fig. 5) and the voltage angles also 
Table 3: Simulated annealing solution - PMU locations.

\begin{tabular}{ccc} 
(20) PMUs & Connexion bus & (3) Connexion PDC \\
\hline 1 & 20 & 1 \\
\hline 2 & 36 & 3 \\
\hline 3 & 19 & 2 \\
\hline 4 & 26 & 2 \\
\hline 5 & 35 & 2 \\
\hline 6 & 25 & 2 \\
\hline 7 & 33 & 2 \\
\hline 8 & 22 & 1 \\
\hline 9 & 2 & 3 \\
\hline 10 & 28 & 3 \\
\hline 11 & 18 & 3 \\
\hline 12 & 38 & 1 \\
\hline 13 & 30 & 2 \\
\hline 14 & 6 & 1 \\
\hline 15 & 21 & 3 \\
\hline 16 & 3 & 1 \\
\hline 17 & 27 & 1 \\
\hline 18 & 17 & 1 \\
\hline 19 & 37 & 1 \\
\hline 20 & 16 & 1 \\
\hline
\end{tabular}

Table 4: Simulated annealing solution - PDC locations.

\begin{tabular}{cc} 
PDC & Connexion bus \\
\hline 1 & 4 \\
\hline 2 & 32 \\
\hline 3 & 14
\end{tabular}


increase (see Fig. 6). On all the figures, it can be observed that both the generator and the grid states are accurately estimated after the initial transients limited to a $150 \mathrm{~ms}$ duration. The observer reacts very well to the load disturbance occuring at $t=200 \mathrm{~ms}$ by still providing an accurate estimate of the state variables despite measurement noise. The system appears to be clearly unstable, since there is no frequency control mechanism in this case study.

Table 5: EKF data (Index "e" stands for the setpoint state).

\begin{tabular}{cccc}
$R$ & $Q$ & $Q_{0}$ & Initial state estimates \\
\hline $1 e^{-4} I_{40 \times 40}$ & $1 e^{-2} I_{40 \times 40}$ & $I_{40 \times 40}$ & $\left(x_{e}^{T}, z_{e}^{T}\right)^{T} \times 0.8$ \\
\hline
\end{tabular}

\section{Conclusions and perspectives}

In this paper, a methodology for designing an optimal monitoring architecture using a limited number of PMUs has been proposed. The optimal design problem consists in maximizing the observability index of the power system (which is based on the trace of the linear observability gramian), while minimizing the communication infrastructure cost. Simulated annealing was sucessfully applied to solve the optimal location problem for a realistic 39 bus system test. Furthermore, a nonlinear dynamical EKF-like state-observer has been proposed, which does not require any on-line "nonlinear inversion" technique. Further researches will be devoted to the design of a distributed version of the here-proposed EKF state observer suitable for both reducing the computational cost and for taking advantage of the distributed nature of wide-area power systems. It would be also very convenient for large commmunication delays to derive some real-time state estimators based on delayed measurements. For that purpose, the theory of time-delay systems is needed. This approach was not investigated in the present paper. It also represents an important research perspective for future works.

\section{References}

Akyol, B.A., Kirkham, H., Clements, S.L., and Hadley, M.D. (2010). A Survey of Wireless Communications for the Electric Power System. Report for the U.S. Department of Energy, 2009. 


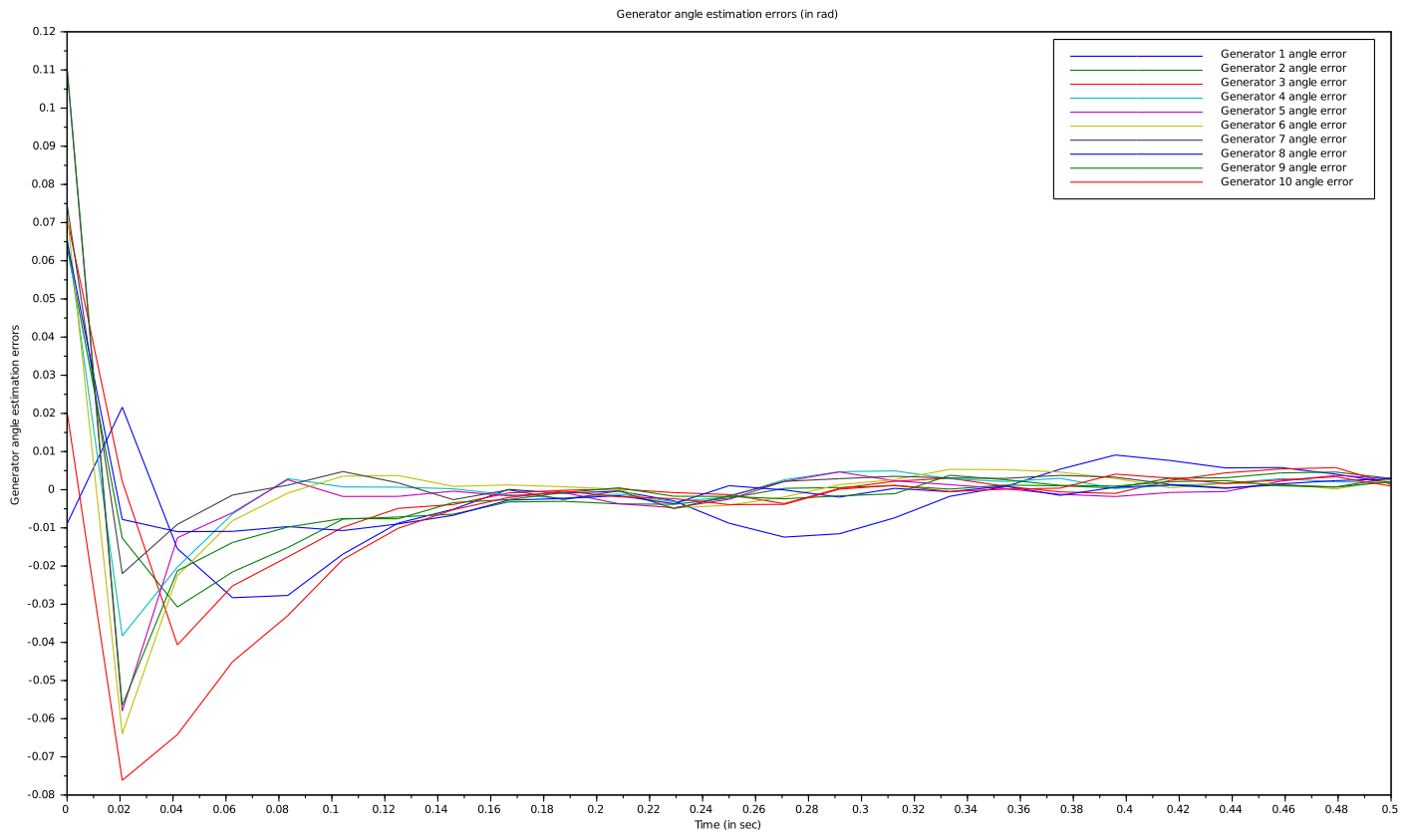

Figure 3: 10-generator angle estimation errors. 


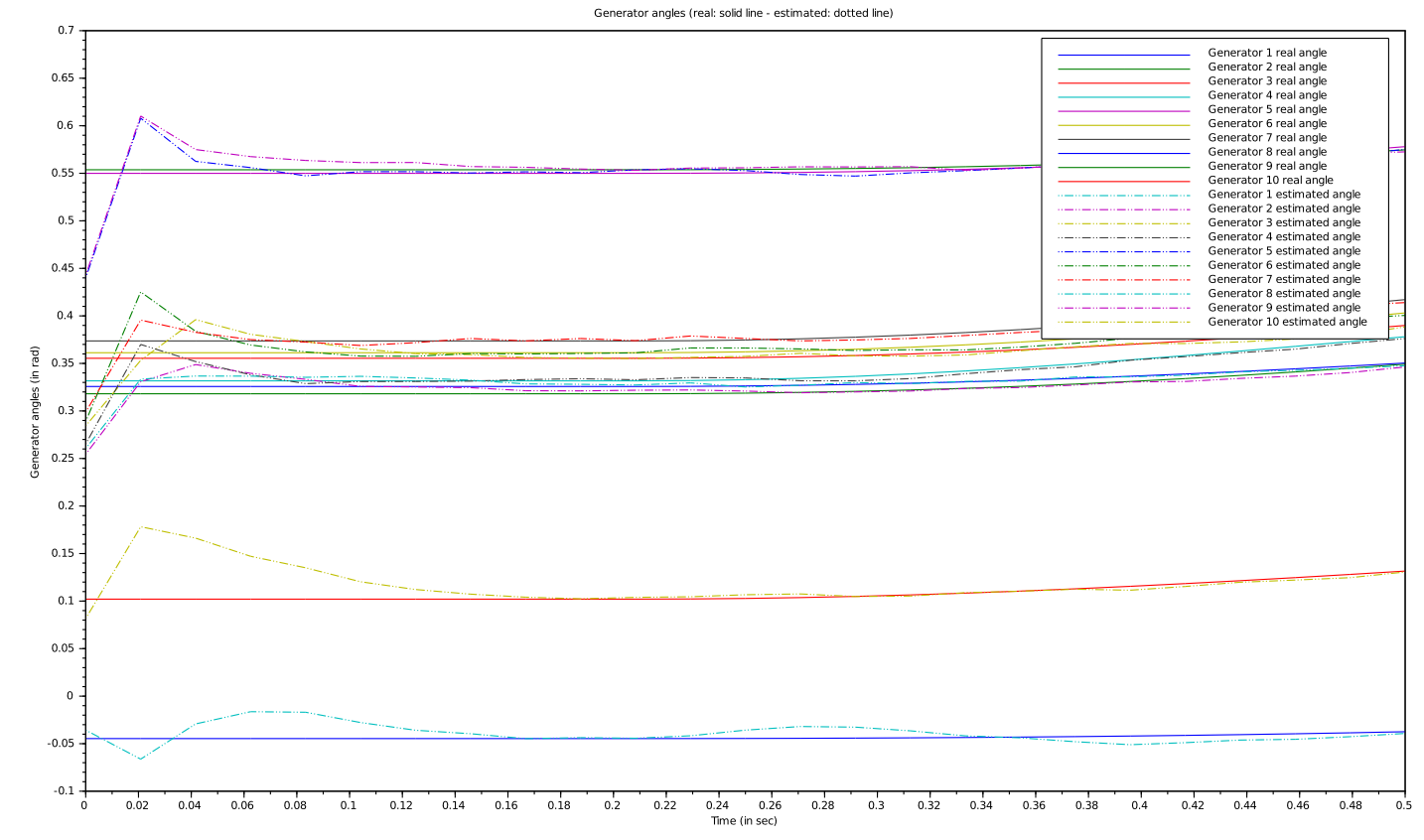

Figure 4: Estimation of the 10-generator angles. 


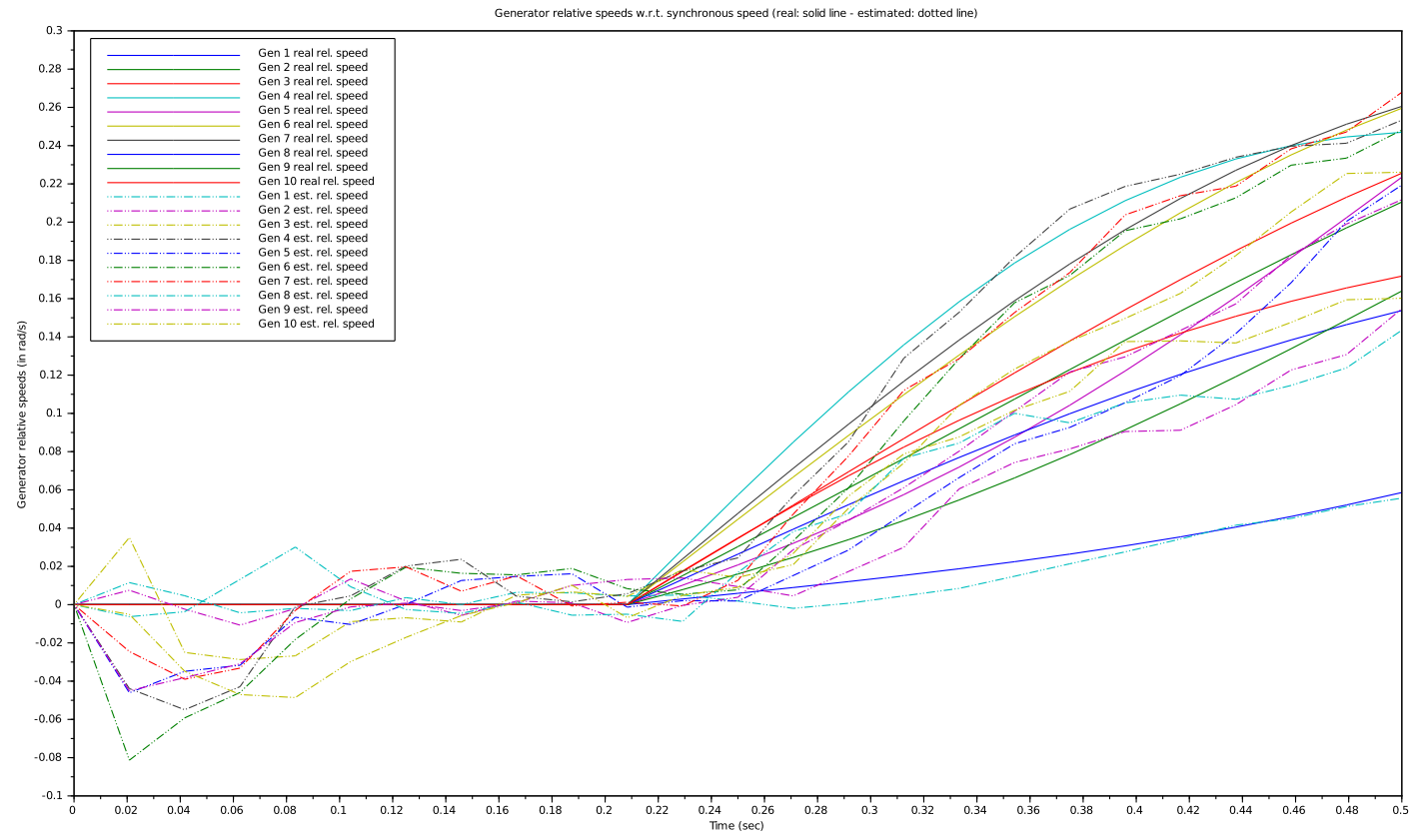

Figure 5: Estimation of the 10-generator relative speeds (w.r.t. synchronous speed $\omega_{0}$ ). 


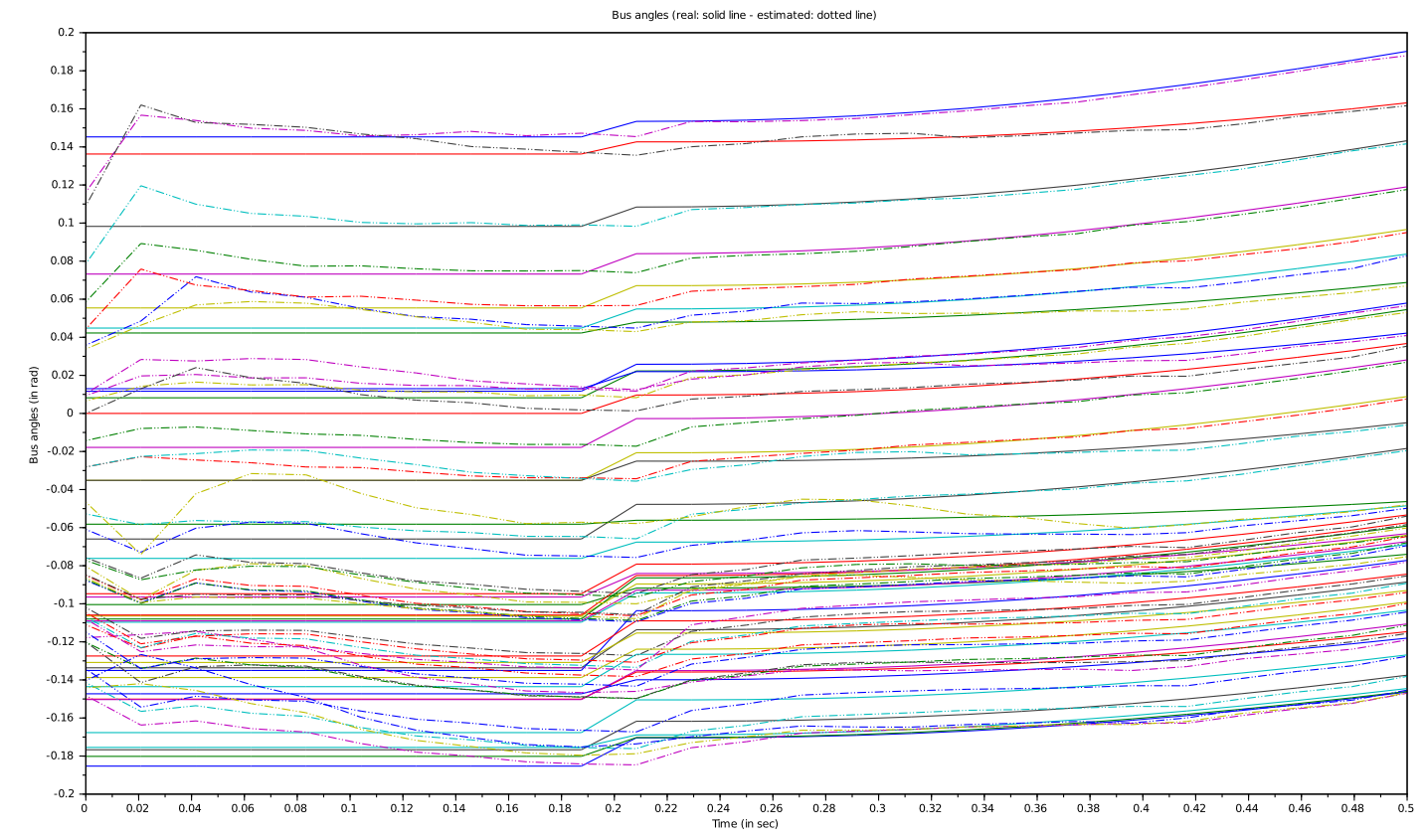

Figure 6: Estimation of the 39-bus voltage angles. 
Baldwin, T., Mili, L., Boisen, J.,and Adapa, R. (1993). Power system observability with minimal phasor measurement placement. IEEE Trans. on Power Systems, vol. 8, no. 2, pp. 707-715, 1993.

Bergen, A.R., and Vittal, V. (2000). Power system analysis. Second edition. Prentice Hall, 2000.

Georges, D. (1995). The use of observability and controllability gramians or functions for optimal sensor and actuator location in finite-dimensional systems. Proceedings of the 34th IEEE Conference on Decision and Control, 1995.

Georges, D. (2011). Energy Minimization and Observability Maximization in Multi-Hop Wireless Sensor Networks. Proceedings of the 18th IFAC World Congress, Milano, Italy, 2011.

Georges, D. (2012). Optimal PMU-based monitoring architecture design for power systems. Proceedings of the IFAC Workshop PPPSC, Toulouse, france, sept. 2012.

Gibbs, B. (2011). Advanced Kalman filtering, least-squares and modeling. J. Wiley and Sons, Inc. 2011.

Gou, B. (2008). Generalized integer linear programming formulation for optimal pmu placement. IEEE Trans. on Power Systems, vol. 23, no. 3, pp. 1099-1104, 2008.

Granville, V., Krivanek, M., Rasson, J.-P. (1994). Simulated annealing: A proof of convergence. IEEE Transactions on Pattern Analysis and Machine Intelligence 16 (6): 652-656, 1994.

Hadjsaid, N., V., Georges, D., Snyder, A. (2008). Applications of Synchronized Phasor Measurements to large interconnected electric power systems. In Electric Power Systems edited by M. Crappe, ISTE ltd, John Wiley \& Sons, Inc., 2008.

Huang, Z., Schneider, K., and Nieplocha, J. (2007). Feasibility studies of applying Kalman filter techniques to power system dynamic state estimation. Proceedings of the International Power Engineering Conference 2007, 2007. 
Ilic, M., and Zaborszky, J. (2000). Dynamics and control of large electric power systems. John Wiley and Sons, Inc., 2000.

Jaimoukha, I. M., and Kasenally, E. M. (1994). Krylov Subspace Methods for Solving Large Lyapunov Equations. SIAM Journal on Numerical Analysis, Vol. 31, No. 1, pp. 227-251, 1994.

Kirkpatrick, S., Gelatt, C. D., and Vecchi, M. P. (1983). Optimization by Simulated Annealing. Science 220, 671-680, 1983.

Moore, B.C. (1981). Principal Component Analysis in Linear Systems: Controllability, Observability, and Model reduction. IEEE Transactions on Automatic Control, vol. 26, 1, pp. 17-32.

Narendra, K., and Weekes, T. (2008). Phasor Measurement Unit (PMU) Communication Experience in a Utility Environment. Conference on Power Systems, Winnipeg, October 19-21, Cigre, 2008.

Nguyen, D.T., Georges, D., and Tran, Q.T. (2008). An Energy Approach to Optimal Selection of Controllers and Sensors in Power System. International Journal of Emerging Electric Power Systems, vol. 9, 2008, [hal00370850], http://www.bepress.com/ijeeps/vol9/iss6/art2.

Pai, M.A. (1989). Energy Function Analysis for Power System Stability. Kluwer Academic Publishers, Boston, 1989.

Phadke, A., Thorp, J., and Adamiak, M. (1983). A new measurement technique for tracking voltage phasors, local system frequency, and rate of change of frequency. IEEE Trans. on Power Apparatus and Systems, vol. 102, no. 5, pp. 1025-1038, may 1983.

Xu, B., and Abur, A. (2004). Observability analysis and measurement placement for systems with pmus. IEEE PES, 10-13 2004, pp. 943-946, vol.2.

Zhou, J., Salomon, G., and Wu, E. (1999). Balanced realization and model reduction for unstable systems. Int. J. Robust Nonlinear Control, Vol. 9, no. 3, pp. 183-198, 1999. 


\section{List of Figures}

1 Example of the location of 5 PMUs connected to 2 PDCs. . . 12

2 The IEEE 10-generator 39-bus power system. . . . . . . . . . 19

3 10-generator angle estimation errors. . . . . . . . . . . 24

4 Estimation of the 10-generator angles. . . . . . . . . . 25

5 Estimation of the 10-generator relative speeds (w.r.t. syn-

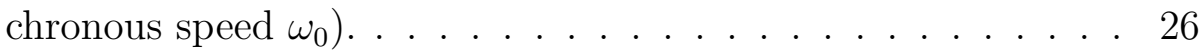

6 Estimation of the 39 -bus voltage angles. . . . . . . . . . . . 27

\section{List of Tables}

1 Enumerative solution versus simulated annealing solution (60 iterations). . . . . . . . . . . . . . . 20

2 Simulated annealing solution (60 iterations). . . . . . . . . 21

3 Simulated annealing solution - PMU locations. . . . . . . . . . 22

4 Simulated annealing solution - PDC locations. . . . . . . . . . 22

$5 \quad$ EKF data (Index "e" stands for the setpoint state). . . . . . . 23 